\title{
A New Nonrelativistic Investigation for the Lowest Excitations States of Interactions in One-Electron Atoms, Muonic, Hadronic and Rydberg Atoms with Modified Inverse Power Potential
}

\author{
Abdelmadjid Maireche \\ Laboratory of Physics and Material Chemistry, Physics department, Sciences Faculty, \\ University of M'sila-M'sila Algeria. \\ E-mail: abmaireche@gmail.com
}

Keywords: The inverse power potential, noncommutative space and phase, star product and Boopp's shift method, muonic atoms, hadronic and Rydberg atoms

\begin{abstract}
A new theoretical analytical investigation for the exact solvability of non-relativistic quantum spectrum systems at low energy for modified inverse power potential (m.i.p.) is discussed by means Boopp's shift method instead to solving deformed Schrödinger equation with star product, in the framework of both noncommutativite two dimensional real space and phase (NC: 2D-RSP), the exact corrections for lowest excitations are found straightforwardly for interactions in oneelectron atoms, muonic, hadronic and Rydberg atoms by means of the standard perturbation theory. Furthermore, the obtained corrections of energies are depended on the four infinitesimals parameters $(\theta, \chi)$ and $(\bar{\theta}, \bar{\sigma})$, which are induced by position-position and momentum-momentum noncommutativity, in addition to the discreet atomic quantum numbers $(j=l \pm 1 / 1, s= \pm 1 / 2, l$ and $m)$ and we have also shown that, the old states are canceled and has been replaced by new degenerated $4(2 l+1)$ sub-states.
\end{abstract}

\section{Introduction}

Over the past few years, many efforts have been produced to study quantum systems on based to the Dirac equation, Klein-Gordon equation and Schrödinger equation, which is undoubtedly the most widely studied equation of modern physics, for spherical and non spherical potentials that are used in different fields of physics and materials sciences by using various methods such as the Nikiforov-Uvarov, super-symmetric quantum mechanics, numerical calculations, asymptotic iteration, the path integral approach, asymptotic iteration method, exact method and many others [125]. Recently, physicists have developed previously works by applying the noncommutativity properties on space and phase, the two ordinary generalized coordinates of space and momentums $\left(x^{\mu}, p^{\mu}\right)$ replaced by corresponding new values $\left(\hat{x}^{\mu}, \hat{p}^{\mu}\right)$, thus the two ordinary commutation relations $\left\lfloor x^{\mu}, x^{v}\right\rfloor$ and $\left\lfloor p^{\mu}, p^{v}\right\rfloor$ will be replace by two new commutation relations in noncommutative quantum mechanics, $\left[\hat{x}^{\mu}, \hat{x}^{v}\right]_{*}$ and $\left[\hat{p}^{\mu}, \hat{p}^{v}\right]_{*}$ respectively, in natural units [26-50]:

$$
\left[\hat{x}^{\mu}, \hat{x}^{v}\right]: \equiv \hat{x}^{\mu} * \hat{x}^{v}-\hat{x}^{v} * \hat{x}^{\mu}=i \theta^{\mu v} \text { and }\left[\hat{p}^{\mu}, \hat{p}^{\nu}\right]: \equiv \hat{p}^{\mu *} \hat{p}^{v}-\hat{p}^{v} * \hat{p}^{\mu}=i \bar{\theta}^{\mu v}
$$

The symbol $(*)$ and the two parameters $\left(\theta^{\mu \nu}, \bar{\theta}^{\mu \nu}\right) \equiv \varepsilon^{\mu v}(\theta, \bar{\theta})$ are star product and antisymmetric real tensors induced by position-position and momentum-momentum noncommutativity, respectively [48], it's important to notice that, the above two fundamental commutation relations are satisfied in particulars' cases from the general star product $f(x, p) * g(x, p)$ between two arbitrary functions $f(x, p)$ and $g(x, p)$ in the first order of two parameters $\theta^{\mu v}$ and $\bar{\theta}^{\mu v}$ as follow [26-44]:

$$
(f * g)(x, p)=(f g)(x, p)-\frac{i}{2} \theta^{\mu \nu} \frac{\partial f(x, p)}{\partial x^{\mu}} \frac{\partial g(x, p)}{\partial x^{v}}-\frac{i}{2} \bar{\theta}^{\mu \nu} \frac{\partial f(x, p)}{\partial p^{\mu}} \frac{\partial g(x, p)}{\partial p^{v}}
$$


The first term is the usual product in commutative space while the rest two parts are represents the effects of the noncommutativity of (space-space) and (phase-phase), respectively; a Boopp's shift method can be used, instead of solving any quantum systems by using directly star product procedure:

$$
\left[\hat{x}_{i}, \hat{x}_{j}\right]=i \theta_{i j} \text { and }\left[\hat{p}_{i}, \hat{p}_{j}\right]=i \bar{\theta}_{i j}
$$

The generalized positions and momentum coordinates in the noncommutative quantum mechanics $(\hat{x}, \hat{y})$ and $\left(\hat{p}_{x}, \hat{p}_{y}\right)$ are depended with corresponding usual generalized positions and momentum coordinates in the usual quantum mechanics $(x, y)$ and $\left(p_{x}, p_{y}\right)$ by the following relations [26-39]:

$$
\begin{aligned}
& \hat{x}=x-\frac{\theta}{2} p_{y}, \quad \hat{y}=y+\frac{\theta}{2} p_{x} \\
& \hat{p}_{x}=p_{x}+\frac{\bar{\theta}}{2} y \text { and } \quad \hat{p}_{y}=p_{x}-\frac{\bar{\theta}}{2} x
\end{aligned}
$$

Which allow us to getting the two operators $\hat{r}^{2}$ and $\hat{p}^{2}$ on a noncommutative two dimensional space-phase as follows [26-39]:

$$
\hat{r}^{2}=r^{2}-\theta L_{z} \quad \text { and } \quad \hat{p}^{2}=p^{2}+\bar{\theta} L_{z}
$$

With $L_{z} \equiv x p_{y}-y p_{x}$, this work is aimed at obtaining an analytic expression for the eigenenergies of a inverse power potential in (NC: 2D-RSP) using the generalization Boopp's shift method based on mentioned formalisms on above equations to discover the new symmetries and a possibility to obtain another applications to this potential in different fields, it is important to notice that, this potential was studied, in ordinary two dimensional spaces, by authors Shi-Hai Dong, Zhoung-Qi Ma, Giam pieero Esposito, E. Vogt and G. H. Wannier of the Refs. [23, 24, 25]. This potential become useful for describes interactions in one-electron atoms, muonic, hadronic and Rydberg atoms; photo decay of excited states, when gaseous ions or electrons move through a gas whose molecules are not too large and we have also for Dirac equation for a spin $1 / 2$ particle interacting with scalar, electric and magnetic potentials leads to second-order equations for spinor wave functions involving an effective potential containing terms proportional to $\frac{1}{r^{2}}, \frac{1}{r^{3}}$ and $\frac{1}{r^{4}}$ as it's mentioned in the refs. $[23,24,25]$. The contents of the rest paper are as follows: In next section, we briefly review the Schrödinger equation with inverse power potential. The Section 3, reserved to derive the deformed potential and perturbative terms by applying both Boopp's shift method to the inverse power potential. In the fourth section and by applying standard perturbation theory we find the quantum spectrum of the lowest excitations in (NC: 2D RSP) for spin-orbital interaction. In the next section, we derive the magnetic spectrum for studied potential. In the sixth section, we resume the global spectrum and corresponding noncommutative Hamiltonian for inverse-power potential. In the seventh section, we extended our study to include the energy spectra for muonic, hadronic and Rydberg atoms in (NC: 2D RSP). Finally, the important results and the conclusions are discussed in last section.

\section{Review the eignenfunctions and the energy eigenvalues for inverse-power potential in ordinary two dimensional spaces}

Let's present a brief review of time independent Schrödinger equation for a fermionic particle like electron of rest mass $m_{0}$ and its energy $E$ moving in inverse power potential $[23,25]$ :

$$
V(r)=A r^{-4}+B r^{-3}+C r^{-2}+D r^{-1}
$$


where $A\rangle 0, D\langle 0$ while $B$ and $C$ are constant coefficients. If we insert this potential into the nonrelativistic Schrödinger equation, we obtain the following equation:

$$
\left(-\frac{1}{2 m_{0}}\left(\frac{1}{r} \frac{\partial}{\partial r}\left(r \frac{\partial}{\partial r}\right)+\frac{1}{r^{2}} \frac{\partial^{2}}{\partial \varphi^{2}}\right)+A r^{-4}+B r^{-3}+C r^{-2}+D r^{-1}\right) \Psi(r, \varphi)=E \Psi(r, \varphi)
$$

here $\Psi(r, \varphi)$ is the solution in the 2-dimensional polar coordinates, the complete wave function $\Psi(r, \varphi)$ separated as follows:

$$
\Psi(\dot{r})=r^{-\frac{1}{2}} R_{m}(r) e^{ \pm i \varphi}
$$

Substituting eq. (8) into eq. (7), we obtain the radial function $R_{m}(r)$ satisfied the following equation, in ordinary two dimensional spaces (2D) [23, 25]:

$$
\left(\frac{d^{2}}{d r^{2}}+E-A r^{-4}-B r^{-3}-C r^{-2}-D r^{-1}-\frac{m^{2}-\frac{1}{4}}{r^{2}}\right) R_{m}(r)=0
$$

here $m$ denote to the orbital angular momentum quantum numbers. The Schrödinger equation given in Eq. (9) has been solved by means of the Nikiforov-Uvarov (NU) method [25], in 2D spaces for inverse power potential for ground state and first excited state, respectively:

$$
\begin{aligned}
& R_{0}(r)=N_{0} r^{c} \exp \left(\frac{a}{r}+b r\right) \\
& R_{1}(r)=N_{1}\left(r-\sigma_{1}^{(1)}\right) r^{c} \exp \left(\frac{a}{r}+b r\right)
\end{aligned}
$$

Where the parameters $\left(a, b, c\right.$ and $\left.\sigma_{1}^{(1)}\right)$ are related with potential coefficients $(A, B, C)$ and $D$ by :

$$
\begin{aligned}
& a^{2}=A, \quad 2 a(1-c)=B, \quad c(c-1)-2 a b=\lambda \\
& 2 b c=D \quad b^{2}=-E, \quad \lambda=\mu(1+\mu)+\frac{D \sqrt{A}}{1+\mu} \quad \mu \frac{B}{\sqrt{4 A}} \\
& \text { and } 2\left(\sigma_{1}^{(1)}-a\right) b^{2}-\lambda b+\frac{1}{2} D c=0
\end{aligned}
$$

It has the two eigenvalues $E_{0}^{ \pm}$and $E_{1}^{ \pm}$(two values for every state), respectively [25]:

$$
\begin{aligned}
& E_{0}^{ \pm}=-\frac{1}{16 A}\left(\lambda \pm \sqrt{\lambda^{2}-2 B D}\right) \\
& E_{1}^{ \pm}=-\left(\frac{\lambda \pm\left(\lambda^{2}-4 D\left(\sigma_{1}^{(1)}+\sqrt{A}\right)(1+\mu)\right)^{1 / 2}}{4\left(\sigma_{1}^{(1)}+\sqrt{A}\right)}\right)
\end{aligned}
$$

The normalized wave functions $\left(\Psi_{0}(r, \varphi), \Psi_{1}(r, \varphi)\right)$ expressed in terms of the radial functions and spherical harmonic functions read as [25]:

$$
\begin{aligned}
& \Psi_{0}(r, \varphi)=N_{0} r^{c-\frac{1}{2}} \exp \left(\frac{a}{r}+b r\right) e^{ \pm i \varphi} \\
& \Psi_{1}(r, \varphi)=N_{1}\left(r-\sigma_{1}^{(1)}\right) r^{c-\frac{1}{2}} \exp \left(\frac{a}{r}+b r\right) e^{ \pm i \varphi}
\end{aligned}
$$


The two normalizations constants $N_{0}$ and $N_{1}$ are given by [25]:

$$
\begin{aligned}
& N_{0}=\left[2\left(\frac{a}{b}\right)^{\frac{2 c+1}{2}} K_{2 c+1}(4 \sqrt{a b})\right]^{-\frac{1}{2}} \\
& N_{1}=\left[2\left(\frac{a}{b}\right)^{\frac{2 c+2}{3}} K_{2 c+3}(4 \sqrt{a b})-4 \sigma_{1}^{(1)}\left(\frac{a}{b}\right)^{c+1} K_{2 c+2}(4 \sqrt{a b})+2\left(\sigma_{1}^{(1)}\right)^{2}\left(\frac{a}{b}\right)^{\frac{2 c+1}{2}} K_{2 c+1}(4 \sqrt{a b})\right]^{-\frac{1}{2}}
\end{aligned}
$$

\section{Theory of two dimensional noncommutative real space-phase (NC-2D: RSP) for (m.i.p.) potential}

In this section, we shall study the (m.i.p.) potential in (NC: 2D-RSP), to perform this task the physical form of non relativistic Schrödinger equation, we apply the following steps [26-44]:

- Ordinary two dimensional Hamiltonian operators $\hat{H}_{i p}\left(p_{i}, x_{i}\right)$ will be replaced by new two dimensional Hamiltonian operators $\hat{H}_{n c-i p}\left(\hat{p}_{i}, \hat{x}_{i}\right)$,

- Ordinary complex wave function $\Psi(\vec{r})$ will be replacing by new complex wave function $\hat{\Psi}(\vec{r})$,

- Ordinary energy $E$ will be replaced by new values $E_{n c-i p}$.

And the last step corresponds to replace the ordinary old product by new star product $(*)$, which allow us to constructing the modified two dimensional Schrödinger equation in both (NC-2D: RSP) as for (m.i.p.) potential:

$$
\hat{H}_{n c-i p}\left(\hat{p}_{i}, \hat{x}_{i}\right) * \hat{\Psi}(\overrightarrow{\vec{r}})=E_{n c-i p} \hat{\Psi}(\overrightarrow{\vec{r}})
$$

It's important to notice that, the two obtained results in eq. (5) conserved the symmetry between $\hat{p}_{i}$ and $\hat{x}_{i}$ and the rolls of $\theta$ and $\bar{\theta}$ are $\operatorname{inversed}(\theta \approx-\bar{\theta})$ [36]. The only none null two commutations relations: $\left\{[\hat{x}, \hat{y}]\right.$ and $\left.\left\{\hat{p}_{x}, \hat{p}_{y}\right]\right\}$ and the two uncertainties relations $\left\{\Delta x \Delta y\right.$ and $\left.\Delta p_{x} \Delta p_{y}\right\}$ are determined from the following projections relations:

$$
\begin{array}{ll}
([\hat{x}, \hat{y}], & \left.\left[\hat{p}_{x}, \hat{p}_{y}\right]\right)=i(\theta,-\bar{\theta}) \\
(\Delta x \Delta y, & \left.\Delta p_{x} \Delta p_{y}\right) \approx(\theta, \bar{\theta})
\end{array}
$$

here $(\theta, \bar{\theta}) \equiv\left(\theta^{12}, \bar{\theta}^{12}\right)$, as mentioned before, we apply the Boopp's shift method on the above equation (11) to obtain the reduced Schrödinger equation for (m.i.p.) potential (without star product):

$$
H\left(\hat{p}_{i}, \hat{x}_{i}\right) \psi(\vec{r})=E_{n c-i p} \psi(\vec{r})
$$

Where the new operator of Hamiltonian $H\left(\hat{p}_{i}, \hat{x}_{i}\right)$ can be expressed in three general varieties: NC2D: RSP (noncommutative space and noncommutative phase), NC: 2D-RSP (only noncommutative space) and NC-2D: RP (only noncommutative phase) as, respectively:

$$
\begin{aligned}
& H_{n c-i p}\left(\hat{p}_{i}, \hat{x}_{i}\right) \equiv H\left(\hat{p}_{x}=p_{x}+\frac{\bar{\theta}}{2} y, \hat{p}_{y}=p_{y}-\frac{\bar{\theta}}{2} x, \hat{x}=x-\frac{\theta}{2} p_{y}, \hat{y}=y+\frac{\theta}{2} p_{x}\right) \text { for } \quad \mathrm{NC}-2 \mathrm{D}: \mathrm{RSP} \\
& H_{n c-i p}\left(\hat{p}_{i}, \hat{x}_{i}\right) \equiv H\left(\hat{p}_{x}=p_{x}, \hat{p}_{y}=p_{y}, \hat{x}=x-\frac{\theta}{2} p_{y}, \hat{y}=y+\frac{\theta}{2} p_{x}\right) \quad \text { for } \quad \mathrm{NC}-2 \mathrm{D}: \mathrm{RS} \\
& H_{n c-i p}\left(\hat{p}_{i}, \hat{x}_{i}\right) \equiv H\left(\hat{p}_{x}=p_{x}+\frac{\bar{\theta}}{2} y, \hat{p}_{y}=p_{x}-\frac{\bar{\theta}}{2} x, \hat{x}=x, \hat{y}=y\right) \quad \text { for } \quad \mathrm{NC}-2 \mathrm{D}: \mathrm{RP}
\end{aligned}
$$


In recently work, we are interest with the first variety (19), after straightforward calculations, we can obtain the five important terms, which will be use to determine the (m.i.p.) potential potential in (NC: 2D- RSP):

$$
\begin{aligned}
& A \hat{r}^{-4}=A r^{-4}+\frac{2 A \theta L_{z}}{r^{6}}, B \hat{r}^{-3}=B r^{-3}+\frac{3 \theta B}{2 r^{5}} L_{z} \\
& C \hat{r}^{-2}=C r^{-2}+\frac{\theta C}{r^{4}} L_{z}, D \hat{r}^{-1}=D r^{-1}+\frac{\theta D}{2 r^{3}} L_{z} \\
& \frac{\hat{p}^{2}}{2 m_{0}}=\frac{p^{2}}{2 m_{0}}+\frac{\bar{\theta}}{2 m_{0}} L_{z}
\end{aligned}
$$

From above relations, one can write the deformed operator $V_{\text {ip }}(\hat{r})$ for inverse power potential and the noncommutative kinetic term $\frac{\hat{p}^{2}}{2 m_{0}}$, respectively:

$$
\begin{aligned}
& V_{\text {ip }}(\hat{r})=A \hat{r}^{-4}+B \hat{r}^{-3}+C \hat{r}^{-2}+D \hat{r}^{-1} \\
& \frac{\hat{p}^{2}}{2 m_{0}}=-\frac{1}{2 m_{0}}\left(\frac{1}{r} \frac{\partial}{\partial r}\left(r \frac{\partial}{\partial r}\right)+\frac{1}{r^{2}} \frac{\partial^{2}}{\partial \varphi^{2}}\right)+\frac{\bar{\theta}}{2 m_{0}} L_{z}
\end{aligned}
$$

Which allow us to obtaining the global potential operator $H_{\text {nc-ip }}(\hat{r})$ for inverse power potential in both (NC: 2D-RSP) as:

$$
H_{\text {nc-ip }}(\hat{r})=A r^{-4}+B r^{-3}+C r^{-2}+D r^{-1}+\left(\theta\left(\frac{2 A}{r^{6}}+\frac{3 B}{2 r^{5}}+\frac{C}{r^{4}}+\frac{D}{2 r^{3}}\right)+\frac{\bar{\theta}}{2 m_{0}}\right) L_{z}
$$

It's clearly, that the four first terms are given the ordinary inverse power potential in 2D space, while the rest terms are proportional's with two infinitesimals parameters ( $\theta$ and $\bar{\theta})$ and then gives the terms of perturbations $H_{\text {per-ip }}(r)$ in (NC: 2D-RSP) as:

$$
H_{\text {per-ip }}(r)=\left(\theta\left(\frac{2 A}{r^{6}}+\frac{3 B}{2 r^{5}}+\frac{C}{r^{4}}+\frac{D}{2 r^{3}}\right)+\frac{\bar{\theta}}{2 m_{0}}\right) L_{z}
$$

\section{The exact spin-orbital spectrum for (m.i.p.) potential in both (NC:2D- RSP) in one-electron atoms:}

Again, the perturbative term $H_{\text {per-ip }}(r)$ can be rewritten to the equivalent physical form for (m.i.p.) potential:

$$
H_{\text {per-ip }}(r)=2\left(\theta\left(\frac{2 A}{r^{6}}+\frac{3 B}{2 r^{5}}+\frac{C}{r^{4}}+\frac{D}{2 r^{3}}\right)+\frac{\bar{\theta}}{2 m_{0}}\right) \vec{S} \vec{L}
$$

The result (18) is logical for two reasons: the first one $\vec{S}=\overrightarrow{1 / 2}$ (in one-electron atoms) and the second $\left(L_{x} \equiv y p_{z}-z p_{y}=0, L_{y} \equiv z p_{x}-x p_{z}=0\right)$, furthermore, the above perturbative term $H_{\text {per-ip }}(r)$ can be rewritten to the following new equivalent form for (m.i.p.) potential:

$$
H_{\text {per-ip }}(r)=\left(\theta\left(\frac{2 A}{r^{6}}+\frac{3 B}{2 r^{5}}+\frac{C}{r^{4}}+\frac{D}{2 r^{3}}\right)+\frac{\bar{\theta}}{2 m_{0}}\right)\left(\vec{J}^{2}-\vec{L}^{2}-\vec{S}^{2}\right)
$$

To the best of our knowledge, we just replace the coupling spin-orbital $\overleftrightarrow{S} \overleftrightarrow{L}$ by the expression $\frac{1}{2}\left(\vec{J}^{2}-\vec{L}^{2}-\overleftrightarrow{S}^{2}\right)$, in quantum mechanics. The set $\left(H_{\text {so-ip }}(r), \mathrm{J}^{2}, \mathrm{~L}^{2}, \mathrm{~S}^{2}\right.$ and $\left.J_{z}\right)$ forms a complete of conserved physics quantities and the eigen-values of the spin orbital coupling operator 
are $k_{ \pm}(j=l \pm 1 / 2, l, s=1 / 2) \equiv \frac{1}{2}\left\{\left(l \pm \frac{1}{2}\right)\left(l \pm \frac{1}{2}+1\right)+l(l+1)-\frac{3}{4}\right\}$ corresponding $j=l+\frac{1}{2}$ (spin up) and $j=l-\frac{1}{2}$ (spin down), respectively, which allows us to form a diagonal $(2 \times 2)$ matrix, with non null elements are $\left(H_{s o-i p}\right)_{11}$ and $\left(H_{s o-i p}\right)_{22}$ for (m.i.p.) potential in (NC: 2D-RSP) as:

$$
\left(H_{s o-i p}\right)_{11}=k_{+}\left(\theta\left(\frac{2 A}{r^{6}}+\frac{3 B}{2 r^{5}}+\frac{C}{r^{4}}+\frac{D}{2 r^{3}}\right)+\frac{\bar{\theta}}{2 m_{0}}\right) \text { if } j=l+\frac{1}{2} \Rightarrow \text { spin - up }
$$

and

$$
\left(H_{s o-i p}\right)_{22}=k_{-}\left(\theta\left(\frac{2 A}{r^{6}}+\frac{3 B}{2 r^{5}}+\frac{C}{r^{4}}+\frac{D}{2 r^{3}}\right)+\frac{\bar{\theta}}{2 m_{0}}\right) \text { if } j=l-\frac{1}{2} \Rightarrow \text { spin - down }
$$

Substituting eq. (8) into (13.a), the radial part of the modified Schrödinger equation, satisfying the following important equation:

$$
\left(\frac{d^{2}}{d r^{2}}+E-A r^{-4}-B r^{-3}-C r^{-2}-D r^{-1}-\left(\theta\left(\frac{2 A}{r^{6}}+\frac{3 B}{2 r^{5}}+\frac{C}{r^{4}}+\frac{D}{2 r^{3}}\right)+\frac{\bar{\theta}}{2 m_{0}}\right)\left(\vec{J}^{2}-\vec{L}^{2}-\overleftrightarrow{S}^{2}\right)-\frac{m^{2}-\frac{1}{4}}{r^{2}}\right) R_{m}(r)=0
$$

Ii is clearly that the above equation including equation (19), the perturbative terms of Hamiltonian operator, which we are subject of discussion in next sub-section, we look for a possibility to getting an exact solution of equation (29) including the added new part $H_{\text {per-ip }}(r)$, which presented by equation (26).

\section{4-1 The exact spin-orbital spectrum for (m.i.p.) potential in both (NC: 2D- RSP) for ground states in one-electron atoms:}

In this sub section, we are going to study the modifications to the energy levels for ground states ( $E_{\mathrm{u} 0-\mathrm{ip}}$ and $\left.E_{\mathrm{d} 0 \text {-ip }}\right)$ for spin up and spin down, respectively, at first order of two parameters ( $\theta$ and $\bar{\theta}$ ) obtained by applying the standard perturbation theory, using eqs. (13) and (29), because the added new part $H_{\text {per-ip }}(r)$ is very small compared to the principal part of inverse power potential (8):

$$
\begin{aligned}
& E_{\text {u0-ip }}=2 \Pi\left|N_{0}\right|^{2} k_{+}(j=l+1 / 2, l, s=1 / 2) \int_{0}^{+\infty} r^{2 c} \exp \left(\frac{2 a}{r}+2 b r\right)\left(\theta\left(\frac{2 A}{r^{6}}+\frac{3 B}{2 r^{5}}+\frac{C}{r^{4}}+\frac{D}{2 r^{3}}\right)+\frac{\bar{\theta}}{2 m_{0}}\right) d r \\
& E_{\text {d0-ip }}=2 \Pi\left|N_{0}\right|^{2} k_{-}(j=l-1 / 2, l, s=1 / 2) \int_{0}^{+\infty} r^{2 c} \exp \left(\frac{2 a}{r}+2 b r\right)\left(\theta\left(\frac{2 A}{r^{6}}+\frac{3 B}{2 r^{5}}+\frac{C}{r^{4}}+\frac{D}{2 r^{3}}\right)+\frac{\bar{\theta}}{2 m_{0}}\right) d r
\end{aligned}
$$

A direct simplification gives:

$$
\begin{aligned}
& E_{\mathrm{u} 0-\mathrm{ip}}=2 \Pi\left|N_{0}\right|^{2} k_{+}(j=l+1 / 2, l, s=1 / 2)\left\{\theta \sum_{\mu=1}^{4} I_{0-\mu}^{s}+\frac{\bar{\theta}}{2 m_{0}} I_{0-5}^{p}\right\} \\
& E_{\mathrm{d} 0-\mathrm{ip}}=2 \Pi\left|N_{0}\right|^{2} k_{-}(j=l-1 / 2, l, s=1 / 2)\left\{\theta \sum_{\mu=1}^{4} I_{0-\mu}^{s}+\frac{\bar{\theta}}{2 m_{0}} I_{0-5}^{p}\right\}
\end{aligned}
$$

Where, the five terms $I_{0-\mu}^{s}(i=\overline{1,4})$ and $I_{0-5}^{p}$ are given by:

$$
\begin{aligned}
& I_{0-1}^{s}=2 A \int_{0}^{+\infty} r^{(2 c-5)-1} \exp \left(\frac{2 a}{r}+2 b r\right) d r, I_{0-2}^{s}=\frac{3 B}{2} \int_{0}^{+\infty} r^{(2 c-4)-1} \exp \left(\frac{2 a}{r}+2 b r\right) d r \\
& I_{0-3}^{s}=C \int_{0}^{+\infty} r^{(2 c-3)-1} \exp \left(\frac{2 a}{r}+2 b r\right) d r, I_{0-4}^{s}=\frac{D}{2} \int_{0}^{+\infty} r^{(2 c-2)-1} \exp \left(\frac{2 a}{r}+2 b r\right) d r \\
& I_{0-5}^{p}=\int_{0}^{+\infty} r^{(2 c-2)-1} \exp \left(\frac{2 a}{r}+2 b r\right) d r
\end{aligned}
$$


Know we apply the special integral [51]:

$$
\int_{0}^{+\infty} r^{v-1} \exp \left(-\left(\frac{\lambda_{2}}{r}+\lambda_{1} r\right)\right) d r=2\left(\frac{\lambda_{2}}{\lambda_{1}}\right)^{\frac{v}{2}} K_{v}\left(2 \sqrt{\lambda_{1} \lambda_{2}}\right)
$$

Where $\left(\lambda_{1}\right.$ and $\left.\lambda_{2}\right)$ are positive numbers and $\left|2 \sqrt{\lambda_{1} \lambda_{2}}\right|<\frac{\pi}{2}$ and $K_{v}$ the modified function of second kind and $\operatorname{order} v$. After straightforward calculations, we can obtain the explicitly results:

$$
\begin{aligned}
& I_{0-1}^{s}=4 A\left(\frac{a}{b}\right)^{\frac{2 c-5}{2}} K_{2 c-5}(4 \sqrt{a b}), I_{0-2}^{s}=3 B\left(\frac{a}{b}\right)^{c-2} K_{c-2}(4 \sqrt{a b}) \\
& I_{0-3}^{s}=2 C\left(\frac{a}{b}\right)^{\frac{2 c-3}{2}} K_{2 c-3}(4 \sqrt{a b}), I_{0-4}^{s}=\frac{D}{2}\left(\frac{a}{b}\right)^{c-1} K_{c-1}(4 \sqrt{a b}) \\
& I_{0-5}^{p}=2\left(\frac{a}{b}\right)^{\frac{2 c+1}{2}} K_{2 c+1}(4 \sqrt{a b})
\end{aligned}
$$

Which allow us to obtaining the exact modifications of fundamental states ( $E_{\mathrm{u} 0 \text {-ip }}$ and $\left.E_{\mathrm{d} 0-\mathrm{ip}}\right)$ produced by spin-orbital effect:

$$
\begin{aligned}
& E_{\mathrm{u} 0 \text {-ip }}=2 \prod\left[2\left(\frac{a}{b}\right)^{\frac{2 c+1}{2}} K_{2 c+1}(4 \sqrt{a b})\right]^{-1} k_{+}(j=l+1 / 2, l, s=1 / 2)\left\{\theta I_{n c 0-s i p}+\frac{\bar{\theta}}{m_{0}} I_{n c 0-p i p}\right\} \\
& E_{\mathrm{d} 0-\text { ip }}=2 \prod\left[2\left(\frac{a}{b}\right)^{\frac{2 c+1}{2}} K_{2 c+1}(4 \sqrt{a b})\right]^{-1} k_{-}(j=l-1 / 2, l, s=1 / 2)\left\{\theta I_{n c 0-s i p}+\frac{\bar{\theta}}{m_{0}} I_{n c 0-p i p}\right\}
\end{aligned}
$$

Where we have introduced new parameters $I_{n c 0-s i p}$ and $I_{n c 0-p i p}$ for the sake of simplicity:

$$
\begin{gathered}
I_{n c 0-s i p} \equiv\left(\frac{a}{b}\right)^{\frac{2 c-1}{2}} K_{2 c-1}(4 \sqrt{a b})\left(\begin{array}{c}
4 A\left(\frac{a}{b}\right)^{\frac{2 c-5}{2}} K_{2 c-5}(4 \sqrt{a b})+3 B\left(\frac{a}{b}\right)^{c-2} K_{c-2}(4 \sqrt{a b})+ \\
2 C\left(\frac{a}{b}\right)^{\frac{2 c-3}{2}} K_{2 c-3}(4 \sqrt{a b})+\frac{D}{2}\left(\frac{a}{b}\right)^{c-1} K_{c-1}(4 \sqrt{a b})
\end{array}\right) \\
I_{n c 0-p i p} \equiv 2\left(\frac{a}{b}\right)^{\frac{2 c+1}{2}} K_{2 c+1}(4 \sqrt{a b})
\end{gathered}
$$

The two terms $\left(I_{n c 0-s i p}, I_{n c 0-p i p}\right)$ are produced with the effect of noncommutative geometry of space and the noncommutativity geometry of phases.

\section{4-2 The exact spin-orbital spectrum modifications for (m.i.p.) potential in both (NC: 2D- RSP)} for first excited states in one-electron atoms:

Now, we turn to the modifications to the energy levels for first excited states ( $E_{\mathrm{ul} \text {-ip }}$ and $\left.E_{\mathrm{d} 1 \text {-ip }}\right)$ for spin up and spin down, respectively, at first order of two parameters ( $\theta$ and $\bar{\theta}$ ), which obtained by applying the standard perturbation theory:

$$
\begin{aligned}
& E_{\mathrm{ulip}}=2 \Pi\left|N_{1}\right|^{2} k_{+}(j=l+1 / 2, l, s=1 / 2) \int_{0}^{+\infty}\left(r^{2 c+2}-2 \sigma_{1}^{(1)} r^{2 c+1}+\left(\sigma_{1}^{(1)}\right)^{2} r^{2 c}\right) \exp \left(\frac{2 a}{r}+2 b r\right)\left(\theta\left(\frac{2 A}{r^{6}}+\frac{3 B}{2 r^{5}}+\frac{C}{r^{4}}+\frac{D}{2 r^{3}}\right)+\frac{\bar{\theta}}{2 m_{0}}\right) d r \\
& E_{\mathrm{d} 1 \text {-ip }}=2 \Pi\left|N_{1}\right|^{2} k_{-}(j=l-1 / 2, l, s=1 / 2) \int_{0}^{+\infty}\left(r^{2 c+2}-2 \sigma_{1}^{(1)} r^{2 c+1}+\left(\sigma_{1}^{(1)}\right)^{2} r^{2 c}\right) \exp \left(\frac{2 a}{r}+2 b r\right)\left(\theta\left(\frac{2 A}{r^{6}}+\frac{3 B}{2 r^{5}}+\frac{C}{r^{4}}+\frac{D}{2 r^{3}}\right)+\frac{\bar{\theta}}{2 m_{0}}\right) d r
\end{aligned}
$$


A direct simplification gives:

$$
\begin{aligned}
& E_{\text {ul-ip }}=2 \prod\left|N_{1}\right|^{2} k_{+}(j=l+1 / 2, l, s=1 / 2)\left\{\theta \sum_{\mu=1}^{12} I_{1-\mu}^{s}+\frac{\bar{\theta}}{2 m_{0}} \sum_{\mu=13}^{15} I_{1-\mu}^{p}\right\} \\
& E_{\text {d1-ip }}=2 \prod\left|N_{1}\right|^{2} k_{-}(j=l-1 / 2, l, s=1 / 2)\left\{\theta \sum_{\mu=1}^{12} I_{1-\mu}^{s}+\frac{\bar{\theta}}{2 m_{0}} \sum_{\mu=13}^{15} I_{1-\mu}^{p}\right\}
\end{aligned}
$$

Where, the 15- terms: $I_{1-\mu}^{s}-(\mu=\overline{1,12})$-and $I_{1-\mu}^{p}-(\mu=\overline{13,15})$-are given by:

$$
\begin{aligned}
& I_{1-1}^{s}=2 A \int_{0}^{+\infty} r^{(2 c-3)-1}-\exp \left(\frac{2 a}{r}+2 b r\right) d r, I_{1-2}^{s}=\frac{3 B}{2} \int_{0}^{+\infty} r^{(2 c-2)-1} \exp \left(\frac{2 a}{r}+2 b r\right) d r \\
& I_{1-3}^{s}= C \int_{0}^{+\infty} r^{(2 c-1)-1} \exp \left(\frac{2 a}{r}+2 b r\right) d r, I_{1-4}^{s}=D \int_{0}^{+\infty} r^{(2 c+1)-1} \exp \left(\frac{2 a}{r}+2 b r\right) d r \\
& I_{1-5}^{s}=-4 A \sigma_{1}^{(1)} \int_{0}^{+\infty} r^{(2 c-4)-1} \exp \left(\frac{2 a}{r}+2 b r\right) d r, I_{1-6}^{s}=-3 \sigma_{1}^{(1)} B \int_{0}^{+\infty} r^{(2 c-3)-1} \exp \left(\frac{2 a}{r}+2 b r\right) d r \\
& I_{1-7}^{s}=-2 \sigma_{1}^{(1)} C \int_{0}^{+\infty} r^{(2 c-2)-1} \exp \left(\frac{2 a}{r}+2 b r\right) d r, I_{1-8}^{s}=-\sigma_{1}^{(1)} D \int_{0}^{+\infty} r^{(2 c-1)-1} \exp \left(\frac{2 a}{r}+2 b r\right) d r \\
& I_{1-9}^{s}=2 A \sigma_{1}^{(1)^{2}} \int_{0}^{+\infty} r^{(2 c-3)-1} \exp \left(\frac{2 a}{r}+2 b r\right) d r, I_{1-10}^{s}=\frac{\left.3 B \sigma_{1}^{(1)}\right)^{2}}{2} \int_{0}^{+\infty} r^{(2 c-2)-1} \exp \left(\frac{2 a}{r}+2 b r\right) d r \\
& I_{1-11}^{s}=C \sigma_{1}^{(1)^{2}} \int_{0}^{+\infty} r^{(2 c-1)-1} \exp \left(\frac{2 a}{r}+2 b r\right) d r, I_{1-12}^{s}=\frac{D \sigma_{1}^{(1)^{2}}}{2} \int_{0}^{+\infty} r^{2 c-1} \exp \left(\frac{2 a}{r}+2 b r\right) d r \\
& I_{1-13}^{p}=\int_{0}^{+\infty} r^{(2 c+3)-1} \exp \left(\frac{2 a}{r}+2 b r\right) d r, I_{1-14}^{p}=-2 \sigma_{1}^{(1)} \int_{0}^{+\infty} r^{(2 c+2)-1} \exp \left(\frac{2 a}{r}+2 b r\right) d r \\
& I_{1-15}^{p}=\sigma_{1}^{(1)^{2}} \int_{0}^{+\infty} r^{(2 c+1)-1} \exp \left(\frac{2 a}{r}+2 b r\right) d r
\end{aligned}
$$

Now we apply the special integral which represents by eq. (35) to obtain the explicitly results:

$$
\begin{aligned}
& I_{1-1}^{s}= 4 A\left(\frac{a}{b}\right)^{\frac{2 c-3}{2}} K_{2 c-3}(4 \sqrt{a b}), I_{1-2}^{s}=3 B\left(\frac{\lambda_{2}}{\lambda_{1}}\right)^{c-1} K_{2 c-2}(4 \sqrt{a b}) \\
& I_{1-3}^{s}= 2 C\left(\frac{\lambda_{2}}{\lambda_{1}}\right)^{\frac{2 c-1}{2}} K_{2 c-1}(4 \sqrt{a b}), I_{1-4}^{s}=2 D\left(\frac{\lambda_{2}}{\lambda_{1}}\right)^{\frac{2 c+1}{2}} K_{2 c+1}(4 \sqrt{a b}) \\
& I_{1-5}^{s}=-8 A \sigma_{1}^{(1)}\left(\frac{a}{b}\right)^{c-2} K_{2 c-4}(4 \sqrt{a b}), I_{1-6}^{s}=-6 \sigma_{1}^{(1)} B\left(\frac{a}{b}\right)^{\frac{2 c-3}{2}} K_{2 c-3}(4 \sqrt{a b}) \\
& I_{1-7}^{s}=-4 \sigma_{1}^{(1)} C\left(\frac{a}{b}\right)^{c-1} K_{2 c-2}(4 \sqrt{a b}), I_{1-8}^{s}=-2 \sigma_{1}^{(1)} D\left(\frac{\lambda_{2}}{\lambda_{1}}\right)^{\frac{2 c-1}{2}} K_{2 v-1}(4 \sqrt{a b}) \\
& I_{1-9}^{s}= 4 A\left(\sigma_{1}^{(1)}\right)^{2}\left(\frac{a}{b}\right)^{\frac{2 c-3}{2}} K_{2 c-3}(4 \sqrt{a b}), I_{1-10}^{s}=3 B\left(\sigma_{1}^{(1)}\right)^{2}\left(\frac{a}{b}\right)^{c-1} K_{2 c-2}(4 \sqrt{a b}) \\
& I_{1-11}^{s}= 2 C\left(\sigma_{1}^{(1)}\right)^{2}\left(\frac{a}{b}\right)^{\frac{2 c-1}{2}} K_{2 c-1}(4 \sqrt{a b}), I_{1-12}^{s}=2 D\left(\sigma_{1}^{(1)}\right)^{2}\left(\frac{a}{b}\right)^{\frac{2 c-1}{2}} K_{2 c-1}(4 \sqrt{a b}) \\
& I_{1-13}^{p}=2\left(\frac{a}{b}\right)^{\frac{2 c+3}{2}} K_{2 c+3}(4 \sqrt{a b}), I_{1-14}^{p}=-4 \sigma_{1}^{(1)}\left(\frac{a}{b}\right)^{c+1} K_{2 c+2}(4 \sqrt{a b}) \\
& I_{1-15}^{p}=2 \sigma_{1}^{(1)^{2}}\left(\frac{a}{b}\right)^{\frac{2 c+1}{2}} K_{2 c+1}(4 \sqrt{a b})
\end{aligned}
$$


Which allow us to obtaining the exact modifications $\left(E_{\text {ul-ip }}\right.$ and $\left.E_{\text {dl-ip }}\right)$ of degenerated first excited states produced for spin-orbital effect:

$$
\begin{aligned}
& E_{\mathrm{ul}-\mathrm{ip}}=2 \prod\left[2\left(\frac{a}{b}\right)^{\frac{2 c+2}{3}} K_{2 c+3}(4 \sqrt{a b})-4 \sigma_{1}^{(1)}\left(\frac{a}{b}\right)^{c+1} K_{2 c+2}(4 \sqrt{a b})+2\left(\sigma_{1}^{(1)}\right)^{2}\left(\frac{a}{b}\right)^{\frac{2 c+1}{2}} K_{2 c+1}(4 \sqrt{a b})\right]^{-1} k_{+}(j=l+1 / 2, l, s=1 / 2)\left\{\theta I_{n c 1-s i p}+\frac{\bar{\theta}}{2 m_{0}} I_{n c 1-p i p}\right\} \\
& E_{\mathrm{dl}-\mathrm{ip}}=2 \prod\left[2\left(\frac{a}{b}\right)^{\frac{2 c+2}{3}} K_{2 c+3}(4 \sqrt{a b})-4 \sigma_{1}^{(1)}\left(\frac{a}{b}\right)^{c+1} K_{2 c+2}(4 \sqrt{a b})+2\left(\sigma_{1}^{(1)}\right)^{2}\left(\frac{a}{b}\right)^{\frac{2 c+1}{2}} K_{2 c+1}(4 \sqrt{a b})\right]^{-1} k_{-}(j=l-1 / 2, l, s=1 / 2)\left\{\theta L_{n c 1-s i p}+\frac{\bar{\theta}}{m_{0}} L_{n c 1-p i p}\right\}
\end{aligned}
$$

Where the two factors $I_{n c 1-s i p}$ and $I_{n c 1-p i p}$ are given by:

$$
I_{n c 1-s i p} \equiv \sum_{\mu=1}^{12} I_{1-\mu}^{s} \quad \text { and } \quad I_{n c 1-p i p} \equiv \sum_{\mu=13}^{15} I_{1-\mu}^{p}
$$

\section{The exact magnetic spectrum for (m.i.p.) potential in both (NC:2D- RSP):}

\section{5-1 The exact magnetic spectrum for (m.i.p.) potential in both (NC: 2D RSP) for ground states in one-electron atoms:}

On the other hand, we consider interested physically meaningful phenomena, which produced automatically from the perturbative terms of modified inverse-power potential related to the influence of an external uniform magnetic field, it's sufficient to apply the following three replacements to describing these phenomena:

$$
\begin{aligned}
\left(\theta\left(\frac{2 A}{r^{6}}+\frac{3 B}{2 r^{5}}+\frac{C}{r^{4}}+\frac{D}{2 r^{3}}\right)+\frac{\bar{\theta}}{2 m_{0}}\right) L_{z} & \rightarrow\left(\chi\left(\frac{2 A}{r^{6}}+\frac{3 B}{2 r^{5}}+\frac{C}{r^{4}}+\frac{D}{2 r^{3}}\right)+\frac{\bar{\sigma}}{2 m_{0}}\right) \vec{B} \vec{L} \\
\theta & \rightarrow \chi B \\
\bar{\theta} & \rightarrow \bar{\sigma} B
\end{aligned}
$$

here $\chi$ and $\bar{\sigma}$ are infinitesimal real proportional's constants, and we choose the magnetic field $\overleftrightarrow{B}=B \vec{k}$, which allow us to introduce the modified new magnetic Hamiltonian $H_{m-i p}$ in (NC: $2 \mathrm{D}$ RSP) as:

$$
H_{m-i p}=\left(\chi\left(\frac{2 A}{r^{6}}+\frac{3 B}{2 r^{5}}+\frac{C}{r^{4}}+\frac{D}{2 r^{3}}\right)+\frac{\bar{\sigma}}{2 m_{0}}\right)(\vec{B} \vec{J}-\overleftrightarrow{S} \overleftrightarrow{B})
$$

where $(-\overleftrightarrow{S} \overleftrightarrow{B})$ denote to the ordinary Hamiltonian of Zeeman Effect. To obtain the exact noncommutative magnetic modifications of energy $\left(E_{\text {mag } 0 \text { ip }}, E_{\text {mag- } 1 \text { ip }}\right)$ for modified inverse-power potential corresponding the ground states and first excited states, respectively, we make the three translations:

$$
\begin{aligned}
& k_{+} \rightarrow \mathrm{m} \\
& \theta \rightarrow \chi \\
& \bar{\theta} \rightarrow \bar{\sigma}
\end{aligned}
$$


We insert the values of the parameters given in Eqs. (58), (59) and (60) into Eqs. (37) and (51) to obtain ( $\left.E_{\text {magoip }}, E_{\text {magl lip }}\right)$ as follows:

$$
\begin{gathered}
E_{\text {mag } 0 \text { ip }}=2 \prod\left[2\left(\frac{a}{b}\right)^{\frac{2 c+1}{2}} K_{2 c+1}(4 \sqrt{a b})\right]^{-1} B m\left\{\chi I_{n c 0-s i p}+\frac{\bar{\sigma}}{m_{0}} I_{n c 0-p i p}\right\} \\
E_{\text {mag-lip }}=2 \prod\left[2\left(\frac{a}{b}\right)^{\frac{2 c+2}{3}} K_{2 c+3}(4 \sqrt{a b})-4 \sigma_{1}^{(1)}\left(\frac{a}{b}\right)^{c+1} K_{2 c+2}(4 \sqrt{a b})+2\left(\sigma_{1}^{(1)}\right)^{2}\left(\frac{a}{b}\right)^{\frac{2 c+1}{2}} K_{2 c+1}(4 \sqrt{a b})\right]^{-1} B m\left\{\chi I_{n c 1-s i p}+\frac{\bar{\sigma}}{2 m_{0}} I_{n c 1-p i p}\right\}
\end{gathered}
$$

we have $-l \leq m \leq+l$, which allow us to fixing $(2 l+1)$ values for the orbital angular momentum quantum numbers.

\section{Obtained results}

Let us now resume the eigenenergies of the modified Schrödinger equation obtained in this paper, the total modified energies ( $\left.E_{\text {nc u0-ip }}-E_{\text {nc d0-ip }}\right)$ and $\left(E_{\text {ncul-ip }}-E_{\text {nc d1-ip }}\right)$ of a particle fermionic with spin up and spin down are determined corresponding fundamental states and first excited states, respectively, for modified inverse-power potential in (NC: 2D-RSP), on based to the Eqs. (12), (37), (38), (51), (52), (61) and (62), we obtain the four new values of global energies:

$$
\begin{aligned}
& E_{\text {nc u0-ip }}=-\frac{1}{16 A}\left(\lambda \pm \sqrt{\lambda^{2}-2 B D}\right)+2 \Pi\left|N_{0}\right|^{2} k_{+}\left\{\theta I_{n c 0-s i p}+\frac{\bar{\theta}}{2 m_{0}} I_{n c 0-p i p}\right\}+2 \Pi\left|N_{0}\right|^{2} B m\left\{\chi I_{n c 0-s i p}+\frac{\bar{\sigma}}{m_{0}} I_{n c 0-p i p}\right\} \\
& E_{\text {nc d0-ip }}=-\frac{1}{16 A}\left(\lambda \pm \sqrt{\lambda^{2}-2 B D}\right)+2 \Pi\left|N_{0}\right|^{2} k_{-}\left\{\theta I_{n c 0-s i p}+\frac{\bar{\theta}}{2 m_{0}} I_{n c 0-p i p}\right\}+2 \Pi\left|N_{0}\right|^{2} B m\left\{\chi I_{n c 0-s i p}+\frac{\bar{\sigma}}{m_{0}} I_{n c 0-p i p}\right\}
\end{aligned}
$$

And

$$
\begin{aligned}
& E_{\text {nc u1-ip }}=-\left(\frac{\lambda \pm\left(\lambda^{2}-4 D\left(\sigma_{1}^{(1)}+\sqrt{A}\right)(1+\mu)\right)^{1 / 2}}{4\left(\sigma_{1}^{(1)}+\sqrt{A}\right)}\right)+2 \Pi\left|N_{1}\right|^{2} k_{+}\left\{\theta I_{n c 1-s i p}+\frac{\bar{\theta}}{2 m_{0}} I_{n c 1-p i p}\right\} \\
& +2 \Pi\left|N_{1}\right|^{2} B m\left\{\chi I_{n c 1-s i p}+\frac{\bar{\sigma}}{m_{0}} I_{n c 1-p i p}\right\} \\
& E_{\text {nc d1-ip }}=-\left(\frac{\lambda \pm\left(\lambda^{2}-4 D\left(\sigma_{1}^{(1)}+\sqrt{A}\right)(1+\mu)\right)^{1 / 2}}{4\left(\sigma_{1}^{(1)}+\sqrt{A}\right)}\right) 2 \Pi\left|N_{1}\right|^{2} k_{-}\left\{\theta I_{n c 1-s i p}+\frac{\bar{\theta}}{2 m_{0}} I_{n c 1-p i p}\right\} \\
& +2 \Pi\left|N_{1}\right|^{2} B m\left\{\chi I_{n c 1-s i p}+\frac{\bar{\sigma}}{m_{0}} I_{n c 1-p i p}\right\}
\end{aligned}
$$

On based to the following three observations:

- The original spectrum contain two possible values of energy $E_{0}^{ \pm}$and $E_{1}^{ \pm}$in ordinary two dimensional space corresponding ground states and first excited states ( equation (12)),

- The quantum number $m$ satisfied the interval $-l \leq m \leq+l$, thus we have $(2 l+1)$ values for this quantum number,

- We have also two values for $j=l+\frac{1}{2}$ and $j=l-\frac{1}{2}$.

Thus, we can deduce the important original results, every state in usually two dimensional space for (m.i.p.) potential will be $4(2 l+1)$ sub-states in the symmetry of (NC: 2D-RSP).

It's clearly, that the obtained eigenvalues of energies are real and then the noncommutative 
diagonal Hamiltonian $H_{n c-i p}$ is Hermitian for (m.i.p.) potential, furthermore it's possible to writing the two elements $\left(H_{n c-i p}\right)_{11}$ and $\left(H_{n c-i p}\right)_{22}$ as follows:

$$
\begin{aligned}
& \left(H_{n c-i p}\right)_{11}=-\frac{1}{2 m_{0}}\left(\frac{1}{r} \frac{\partial}{\partial r}\left(r \frac{\partial}{\partial r}\right)+\frac{1}{r^{2}} \frac{\partial^{2}}{\partial \varphi^{2}}\right)+A r^{-4}+B r^{-3}+C r^{-2}+D r^{-1}+\frac{l}{2}\left(\theta\left(\frac{2 A}{r^{6}}+\frac{3 B}{2 r^{5}}+\frac{C}{r^{4}}+\frac{D}{2 r^{3}}\right)+\frac{\bar{\theta}}{2 m_{0}}\right) \\
& +\left(\chi\left(\frac{2 A}{r^{6}}+\frac{3 B}{2 r^{5}}+\frac{C}{r^{4}}+\frac{D}{2 r^{3}}\right)+\frac{\bar{\sigma}}{2 m_{0}}\right)(\overrightarrow{B J}-\overleftrightarrow{S B})
\end{aligned}
$$

and

$$
\begin{aligned}
& \left(H_{n c-i p}\right)_{22}=-\frac{1}{2 m_{0}}\left(\frac{1}{r} \frac{\partial}{\partial r}\left(r \frac{\partial}{\partial r}\right)+\frac{1}{r^{2}} \frac{\partial^{2}}{\partial \varphi^{2}}\right)+A r^{-4}+B r^{-3}+C r^{-2}+D r^{-1}-\frac{l+1}{2}\left(\theta\left(\frac{2 A}{r^{6}}+\frac{3 B}{2 r^{5}}+\frac{C}{r^{4}}+\frac{D}{2 r^{3}}\right)+\frac{\bar{\theta}}{2 m_{0}}\right) \\
& +\left(\chi\left(\frac{2 A}{r^{6}}+\frac{3 B}{2 r^{5}}+\frac{C}{r^{4}}+\frac{D}{2 r^{3}}\right)+\frac{\bar{\sigma}}{2 m_{0}}\right)(\overrightarrow{B J}-\overleftrightarrow{S} \vec{B})
\end{aligned}
$$

On the other hand, the above obtain results (67) and (68) allow constructing the diagonal anisotropic matrixes $\left\lfloor\left(H_{n c-i p}\right)_{11} \neq\left(H_{n c-i p}\right)_{22}\right\rfloor$ of the Hamiltonian operator $H_{n c-i p}$ for (m.i.p.) potential in (NC: 2D-RSP) as:

$$
H_{n c-i p}=\left(\begin{array}{cc}
\left(H_{n c-i p}\right)_{11} & 0 \\
0 & \left(H_{n c-i p}\right)_{22}
\end{array}\right)
$$

Which allows us to obtain the original results for this investigation: the obtained Hamiltonian operator (69) describing atom which has two permanent dipoles: the first is electric dipole moment and the second is magnetic moment in external stationary electromagnetic field.

\section{Generalization the obtained results to the energy spectra for muonic, hadronic and Rydberg atoms $(\overleftrightarrow{S} \neq \overrightarrow{1 / 2})$ :}

As it is mentioned in the introduction, the inverse power potential can be described the interactions in muonic (an electron is replaced by a muon), hadronic (one or more of the orbital electrons is replaced by a charged hadron) and Rydberg atoms; photo decay of excited states, it is possible to discover energy spectra for a system (muonic, hadronic and Rydberg atoms) with spin $\overleftrightarrow{S} \neq \overrightarrow{1 / 2}$ in (NC: 2D-RSP), the atomic quantum number $m$ and $j$ can be takes $(2 l+1)$ values and $\underbrace{j=|l-s|,|l-s|+1, \ldots, \ldots j=|l+s| \text { and } j=|l+s|}_{\text {N-possible valuesfor } \mathrm{j}}$ values, respectively, thus every old state in usually two dimensional space will be $2 N(2 l+1)$ sub-states for (m.i.p.). Furthermore, we can deduce the global noncommutative Hamiltonian matrix $H_{n c-i p}$ in both (NC_2D: RSP) for studied potential on based to the previously obtained results, presented in eqs. (63) and (67), in addition to the usually Hamiltonian operator in eq. (7):

$$
\begin{aligned}
& H_{n c-i p}=-\frac{1}{2 m_{0}}\left(\frac{1}{r} \frac{\partial}{\partial r}\left(r \frac{\partial}{\partial r}\right)+\frac{1}{r^{2}} \frac{\partial^{2}}{\partial \varphi^{2}}\right)+A r^{-4}+B r^{-3}+C r^{-2}+D r^{-1}+k(j, l, s)\left(\theta\left(\frac{2 A}{r^{6}}+\frac{3 B}{2 r^{5}}+\frac{C}{r^{4}}+\frac{D}{2 r^{3}}\right)+\frac{\bar{\theta}}{2 m_{0}}\right) \\
& +\left(\chi\left(\frac{2 A}{r^{6}}+\frac{3 B}{2 r^{5}}+\frac{C}{r^{4}}+\frac{D}{2 r^{3}}\right)+\frac{\bar{\sigma}}{2 m_{0}}\right)(\overrightarrow{B J}-\overleftrightarrow{S} \vec{B})
\end{aligned}
$$

And the corresponding energy spectra ( $E_{\text {nc } 0 \text {-ip }}$ and $E_{\text {nc 1-ip }}$ ) for ground state and first excited state, for a system (muonic, hadronic and Rydberg atoms), respectively:

$$
E_{\text {nc } 0 \text {-ip }}=-\frac{1}{16 A}\left(\lambda \pm \sqrt{\lambda^{2}-2 B D}\right)+2 \Pi\left|N_{0}\right|^{2} k(j, l, s)\left\{\theta I_{n c 0-s i p}+\frac{\bar{\theta}}{2 m_{0}} I_{n c 0-p i p}\right\}+2 \Pi\left|N_{0}\right|^{2} B m\left\{\chi I_{n c 0-s i p}+\frac{\bar{\sigma}}{m_{0}} I_{n c 0-p i p}\right\}
$$




$$
E_{\text {nc 1-ip }}=-\left(\frac{\lambda \pm\left(\lambda^{2}-4 D\left(\sigma_{1}^{(1)}+\sqrt{A}\right)(1+\mu)\right)^{1 / 2}}{4\left(\sigma_{1}^{(1)}+\sqrt{A}\right)}\right)+2 \Pi\left|N_{1}\right|^{2} k_{+}\left\{\theta I_{n c 1-s i p}+\frac{\bar{\theta}}{2 m_{0}} I_{n c 1-p i p}\right\}+2 \Pi\left|N_{1}\right|^{2} B m\left\{\chi I_{n c 1-s i p}+\frac{\bar{\sigma}}{m_{0}} I_{n c 1-p i p}\right\}
$$

We have replaced the factor $k_{ \pm}(j=l \pm 1 / 2, l, s=1 / 2)$ by new values $k(j, l, s)$ which determined from the projection relation:

$$
k(j, l, s) \equiv \frac{1}{2}\{j(j+1)+l(l+1)-s(s+1)\}
$$

\section{Conclusions}

To summarize, this work has been devoted to find the solutions of modified Schrodinger equation for the modified inverse power potential, we have obtained the exact energy spectrum for ground states and first excited states in (NC: 2D-RSP). We shown that the old states were changed radically and replaced by degenerated new states, describing two new original spectrums, the first one, produced by the of spin-orbital interaction while the second new spectrum produced by an external magnetic field, determined by the new results (37), (38), (51), (52), (61) and (62) in addition to the usual spectrum produced by ordinary potential. And we have also shown, every state in usually two dimensional spaces will be $4(2 l+1)$ sub-states in the symmetries of (NC: $2 \mathrm{D}-\mathrm{RSP})$. Furthermore, our recently study can be generalized to including the interactions for muonic, hadronic and Rydberg atoms.

\section{Acknowledgement}

This work was supported with search laboratory of Physics and Material Chemistry, in Physics department, Sciences faculty-University of M'sila, Algeria.

\section{References}

[1] Shi-Hai Dong, Guo-Hua Sun, The Schrödinger equation with a Coulomb plus inverse-square potential in D dimensions, Physica Scripta. 70(2-3) (2004) 94-97.

[2] J.J. Pena, G. Ovando, J. Morales, D-dimensional Eckart+deformed Hylleraas potential: Bound state solutions, Journal of Physics: Conference Series. 574 (2015) 012089.

[3] L. Buragohain, S.A.S .Ahmed, Exactly solvable quantum mechanical systems generated from the anharmonic potentials, Lat. Am. J. Phys. Educ. 4(1) (2010) 79-83.

[4] A. Niknam, A.A. Rajab, M. Solaimani, Solutions of D-dimensional Schrödinger equation for Woods-Saxon potential with spin-orbit, coulomb and centrifugal terms through a new hybrid numerical fitting Nikiforov-Uvarov method, J. Theor. App. Phys. 10(1) (2016) 53-59.

[5] Sameer M. Ikhdair, Ramazan Sever, Exact solutions of the radial Schrödinger equation for some physical potentials, CEJP. 5(4) (2007) 516-527.

[6] M.M. Nieto, Hydrogen atom and relativistic pi-mesic atom in N-space dimension, Am. J. Phys. 47 (1979) 1067-1072.

[7] S. M. Ikhdair, R. Sever, Exact polynomial eigensolutions of the Schrödinger equation for the pseudoharmonic potential, J. Mol. Struc.-Theochem. 806(1) (2007) 155-158.

[8] A.S. Ahmed, L. Buragohain, Generation of new classes of exactly solvable potentials, Phys. Scr. 80 (2009) 1-6.

[9] S.K. Bose, Exact solution of non-relativistic Schrödinger equation for certain central physical potentials, Nouvo Cimento B. 113 (1996) 299- 328.

[10] G.P. Flesses, A. Watt, An exact solution of the Schrödinger equation for a multiterm potential, J. Phys. A: Math. Gen. 14 (19981) L315-L318. 
[11] M. Ikhdair, R. Sever, Exact solution of the Klein-Gordon equation for the PT symmetri generalized Woods-Saxon potential by the Nikiforov-Uvarov method, Ann. Phys. (Leipzig). 16 (2007) 218-232.

[12] S.H. Dong, Schrödinger equation with the potential V(r) $=r^{*}-4+r^{*}-3+r^{*}-2+r^{*}-1$, Physica Scripta. 64(4) (2001) 273-276.

[13] S.H. Dong, Z.Q. Ma, Exact solutions to the Schrödinger equation for the potential V(r) $=r^{*} 2+r^{*}-4+r^{*}-6$ in two dimensions, Journal of Physics A. 31(49) (1998) 9855-9859.

[14] S.H. Dong, A new approach to the relativistic Schrödinger equation with central potential: Ansatz method, International Journal of Theoretical Physics. 40(2) (2001) 559-567.

[15] Ali Akder et al., A new Coulomb ring-shaped potential via generalized parametetric Nikivforov-Uvarov method, Journal of Theoretical and Applied Physics. 7 (2013) 17.

[16] Sameer M. Ikhdair, Ramazan Sever, Relativistic Two-Dimensional Harmonic Oscillator Plus Cornell Potentials in External Magnetic and AB Fields, Advances in High Energy Physics. (2013) Article ID 562959.

[17] Shi-Hai Dong, Guo-Hua San, Quantum Spectrum of Some Anharmonic Central Potentials: Wave Functions Ansatz, Foundations of Physics Letters. 16(4) (2003) 357-367.

[18] L. Buragohain, S. A. S. Ahmed, Exactly solvable quantum mechanical systems generated from the anharmonic potentials, Lat. Am. J. Phys. Educ. 4(1) (2010) 79-83.

[19] S.M. Ikhdair, Exact solution of Dirac equation with charged harmonic oscillator in electric field: bound states, Journal of Modern Physics. 3(2) (2012) 170-179.

[20] H. Hassanabadi et al., Exact solution Dirac equation for an energy-depended potential, Tur. Phys. J. Plus. 127 (2012) 120.

[21] H. Hassanabadi et al., Exact solutions of N-Dimensional Schrödinger equation for a potential containing coulomb and quadratic terms, International Journal of the Physical Sciences. 6(3) (2011) 583-586.

[22] D. Agboola, Complte Analytical Solutions of the Mie-Type Potentials in N-Dimensions, Acta Physica Polonica A. 120 (2011) 371-377.

[23] Dong, S et al., Exact solutions of the Schrödinger equation with inverse-power potential, Foundations of Physics Letters. 12(5) (1999) 465-474.

[24] E. Vogt, G.H. Wannier, Scattering of ions by polarization forces, Phys. Rev. 95 (1954) 11901198.

[25] Shi-Hai Dong, Schrödinger Equation with the Potential V(r) $=$ Ar-4 + Br-3 + Cr-2 + Dr-1; Physica Scripta. 64 (2001) 273-276.

[26] Abdelmadjid Maireche, Spectrum of Schrödinger Equation with H.L.C. Potential in NonCommutative Two-dimensional Real Space, The African Rev. Phys. 9 (2014) 479-483.

[27] Abdelmadjid Maireche, Deformed Quantum Energy Spectra with Mixed Harmonic Potential for Nonrelativistic Schrödinger equation, J. Nano- Electron. Phys. 7(2) (2015) 02003.

[28] Abdelmadjid Maireche, A Study of Schrödinger Equation with Inverse Sextic Potential in 2dimensional Non-commutative Space, The African Rev. Phys. 9 (2014) 185-193.

[29] Abdelmadjid. Maireche, Nonrelativistic Atomic Spectrum for Companied Harmonic Oscillator Potential and its Inverse in both NC-2D: RSP, International Letters of Chemistry, Physics and Astronomy. 56 (2015) 1-9.

[30] Abdelmadjid Maireche, Atomic Spectrum for Schrödinger Equation with Rational Spherical Type Potential in Non-commutative Space and Phase, The African Review of Physics. 10 (2015) 373-381.

[31] Abdelmadjid Maireche, New exact bound states solutions for (C.F.P.S.) potential in the case of Non-commutative three dimensional non relativistic quantum mechanics, Med. J. Model. Simul. 4 (2015) 060-072. 
[32] Abdelmadjid. Maireche, New Exact Solution of the Bound States for the Potential Family $\mathrm{V}(\mathrm{r})=\mathrm{A} / \mathrm{r} 2-\mathrm{B} / \mathrm{r}+\mathrm{Crk}(\mathrm{k}=0,-1,-2)$ in both Noncommutative Three Dimensional Spaces and Phases: Non Relativistic Quantum Mechanics, International Letters of Chemistry, Physics and Astronomy. 58 (20145) 164-176.

[33] Abdelmadjid Maireche, A New Approach to the Non Relativistic Schrödinger equation for an Energy-Depended Potential $V\left(r, E_{n, l}\right)=V_{0}\left(1+\eta E_{n, l}\right) r^{2}$ in Both Noncommutative three Dimensional spaces and phases, International Letters of Chemistry, Physics and Astronomy. 60 (2015) 11 19.

[34] Abdelmadjid Maireche, A Recent Study of Quantum Atomic Spectrum of the Lowest Excitations for Schrödinger Equation with Typical Rational Spherical Potential at Planck's and Nanoscales, J. Nano- Electron. Phys. 7(3) (2015) 02003.

[35] Abdelmadjid Maireche, Quantum Hamiltonian and Spectrum of Schrödinger Equation with companied Harmonic Oscillator Potential and its Inverse in three Dimensional Noncommutative Real Space and Phase, J. Nano- Electron. Phys. 7(4) (2015) 04021-1.

[36] Abdelmadjid Maireche, Spectrum of Hydrogen Atom Ground State Counting Quadratic Term in Schrödinger Equation, The African Rev. Phys. 10 (2015) 177-183.

[37] Abdelmadjid Maireche, New Relativistic Atomic Mass Spectra of Quark ( $\mathrm{u}, \mathrm{d}$ and s) for Extended Modified Cornell Potential in Nano and Plank's Scales, J. Nano- Electron. Phys. 8(1) (2016) 01020.

[38] Abdelmadjid Maireche, The Nonrelativistic Ground State Energy Spectra of Potential Counting Coulomb and Quadratic Terms in Non-commutative Two Dimensional Real Spaces and Phases, J. Nano- Electron. Phys. 8(1) (2016) 01021.

[39] Abdelmadjid Maireche, A New Nonrelativistic Atomic Energy Spectrum of Energy Dependent Potential for Heavy Quarkouniom in Noncommutative Spaces and Phases Symmetries, J. Nano- Electron. Phys. 8(2) (2016) 02046.

[40] H. Hassanabadi, F. Hoseini, S. Zarrinkamar, A generalized interaction in noncommutative space: Both relativistic and nonrelativistic fields, Eur. J. Plus. 130(10) (2015) 1-7.

[41] Abdelmalek Boumali, Hassan Hassanabadi, The thermal properties of a two-dimensional Dirac oscillator under an external magnetic field, Eur. J. Plus. 128(10) (2013) 1-13.

[42] A.E.F. Djemei, H. Smail, On Quantum Mechanics on Noncommutative Quantum Phase Space, Commun. Theor. Phys. (Beijinig, China). 41 (2004) 837-844.

[43] Shaohong Cai, Tao Jing, Guangjie Guo, Rukun Zhang, Dirac Oscillator in Noncommutative Phase Space, International Journal of Theoretical Physics. 49(8) (2010) 1699-1705.

[44] Joohan Lee, Star Products and the Landau Problem, Journal of the Korean Physical Society, 47(4) (2005) 571-576.

[45] A. Jahan, Noncommutative harmonic oscillator at finite temperature: a path integral approach, Brazilian Journal of Physics. 37(4) (2007) 144-146.

[46] Anselme F. Dossa, Gabriel Y. H. Avossevou, Noncommutative Phase Space and the Two Dimensional Quantum Dipole in Background Electric and Magnetic Fields, Journal of Modern Physics. 4 (2013) 1400-1411.

[47] Yang, Zu-Hua et al., DKP Oscillator with spin-0 in Three dimensional Noncommutaive Phase-Space, Int. J. Theor. Phys. 49 (2010) 644-657.

[48] Y. Yuan e al., Spin $1 / 2$ relativistic particle in a magnetic field in $\mathrm{NC} \mathrm{Ph}$, Chinese Physics $\mathrm{C}$, 34(5) (2010) 543.

[49] Jumakari-Mamat; Sayipjamal Dulat, Hekim Mamatabdulla, Landau-like Atomic Proplem on a Non-commutative Phase Space, Int J Theor Phys. 55(6) (2016) 2913-2918.

[50] Behrouz Mirza et al., Relativistic Oscillators in a Noncommutative space in a Magnetic field, Commun. Theor. Phys. 55 (2011) 405-409.

[51] M. Abramowitz, I. A. Stegun, Handbook of Mathematical Functions with Formulas, Graphs and Mathematical Tables, Dover Publications, New York, 1965. 